\title{
Pediatric Patients with SARS-CoV-2 Infection: Clinical Characteristics in the United States from a Large Global Health Research Network
}

\author{
Ankita Desai ${ }^{1}$, Alexandra Mills ${ }^{2}$, Sarah Delozier ${ }^{2}$, Claudia Cabrera Aviles ${ }^{2}$, Amy Edwards ${ }^{1}$, Sahera \\ Dirajlal-Fargo ${ }^{1}$, Grace McComsey ${ }^{1}$
}

1. Pediatric Infectious Diseases, University Hospitals Rainbow Babies and Children's Hospital, Cleveland, USA 2. Center for Clinical Research, University Hospitals Cleveland Medical Center, Cleveland, USA

Corresponding author: Ankita Desai, ankita.desai@uhhospitals.org

\section{Abstract \\ Background}

Few reports have been published on the clinical presentation of pediatric patients infected with severe acute respiratory syndrome coronavirus 2 (SARS-CoV-2). We aim to shed more light on the clinical presentation of pediatric patients infected with coronavirus disease 2019 (COVID-19), and also potential risk factors for more severe clinical case presentation.

\section{Methods}

We used a large global health research network to gather clinical data extracted from the electronic medical records of pediatric patients aged < 18 years with confirmed SARS-CoV-2 from January 1, 2020 to May 7, 2020. Clinical symptoms at presentation, hospitalization status, associated co-morbidities, and treatments received were reviewed.

\section{Results}

A total of 627 patients with COVID-19 diagnosis (334 were outpatient, 293 were inpatient) were included from a total of 20 organizations across the United States. The mean age of patients was seven years, $48 \%$ were females. Inpatients were younger than outpatients (mean age of 5.6 years vs 8.2 years, $p<0.001$ ). Sixtyone percent of patients in the inpatient group were $<5$ years of age vs. $44 \%$ in the outpatient group. Amongst 293 inpatients, 90\% ( $n=265)$ were non-severe and 10\% $(n=28)$ were classified as severe. The percentage of patients $<5$ years was higher in severe inpatients vs. non-severe ( $71 \%$ vs $60 \%$.) Significantly more patients with a severe illness vs. non-severe illness had a history of co-morbidity including non-congenital heart disease $(50 \%$ vs $11 \%, \mathrm{p}<0.001)$ and disease of the respiratory system $(86 \%$ vs $53 \%, \mathrm{p}<0.001)$.

\section{Conclusion}

Received 09/04/2020

Review began 09/06/2020 Review ended 09/09/2020 Published 09/12/2020

\section{() Copyright 2020}

Desai et al. This is an open access article distributed under the terms of the Creative Commons Attribution License CC-BY 4.0., which permits unrestricted use, distribution, and reproduction in any medium, provided the original author and source are credited.
Clinicians should closely monitor young children with underlying conditions and COVID-19, as they may be more likely to be hospitalized and have a higher severity of the disease.

Categories: Pediatrics, Infectious Disease

Keywords: covid-19, sars-cov-2, pediatric

\section{Introduction}

In just the span of a few short months, we were notified of coronavirus disease 2019 (COVID-19), a severe respiratory illness, caused by the novel severe acute respiratory syndrome coronavirus 2 (SARS-CoV-2), with subsequent spread across the globe [1]. While we have gained a tremendous amount of knowledge regarding this virus, transmission, and clinical presentation, we still have much to learn [2]. Perhaps one of the most intriguing questions raised by this virus relates to the impact on children. We are yet to fully understand why children seem to be less susceptible to severe infection compared to adults [3].

In humans, coronaviruses mostly cause respiratory or gastrointestinal illness. Reported clinical manifestations range from the common cold to severe illness with severe acute respiratory distress syndrome and multi-organ failure [4].

Few reports have been published on the clinical presentation of pediatric patients infected with SARS-CoV-2 [1-2,4-7]. Recommendations for treatment of children with COVID-19 are largely extrapolated from adult studies [8]. With this report, we aim to shed more light on the clinical presentation of those pediatric patients infected with COVID-19, and also potential risk factors that may lead to more severe clinical case presentation. We looked at a large database, with specific attention to clinical symptoms at presentation, 
hospitalization status, associated co-morbidities, and treatments received for pediatric patients in the United States.

\section{Materials And Methods}

We used a large global health research network to gather clinical data extracted from the electronic medical records of pediatric patients aged less than 18 years with confirmed SARS-CoV- 2 infection in 20 health care organizations in the United States from January 1, 2020 to May 7, 2020. We were able to differentiate inpatient (hospitalized) subjects from outpatients (those who were never hospitalized). In addition, inpatients were further characterized into severe progression, defined as requiring mechanical ventilation or death, or were otherwise classified as inpatients, non-severe. Clinical information was gathered for demographics, medical diagnoses prior to COVID-19 diagnosis, clinical symptoms and laboratory findings at COVID-19 presentation, and medication history at the time of COVID-19 diagnosis.

Data was analyzed using TriNetX (Cambridge, MA), a global federated health research network providing access to statistics on electronic medical records from approximately 53 million patients in 41 healthcare organizations. As a federated network, TriNetX received a waiver from Western Institutional Review Board. In order to prevent the possibility of patient re-identification the TriNetX platform takes several precautions. Amongst these, no individual patient records are available, and all data are reported as aggregates. TriNetX also rounds all patient counts from $1-10$ as $\leqslant 10$. We report this $1-10$ value range as $\leqslant 10$ and report the exact values of 0 as 0 . In-platform analyses were conducted directly from the TriNetX platform, in which continuous data are presented as means and standard deviations, and inferential analyses were conducted using independent t-tests. Categorical data are presented as frequencies and percentages and inferential analyses were conducted using chi-square tests. Due to the obfuscation of patient numbers to keep the de-identification of data in the network, numbers are rounded thus creating differences within and between cohorts. All tests were two-tailed and $\mathrm{p} \leqslant .05$ considered significant.

\section{Results}

\section{Outpatients vs. inpatients}

For this analysis, 627 patients with COVID-19 diagnosis (334 were outpatient, 293 were inpatient) were included from a total of 20 organizations across the United States (Table 1).

\begin{tabular}{|c|c|c|c|c|c|c|}
\hline Variable & $\begin{array}{l}\text { Outpatient all } \\
(\mathrm{N}=334)\end{array}$ & $\begin{array}{l}\text { Inpatient all } \\
(N=293)\end{array}$ & $\begin{array}{l}\text { P- } \\
\text { value }\end{array}$ & $\begin{array}{l}\text { Inpatient non- } \\
\text { severe }(\mathrm{N}=265)\end{array}$ & $\begin{array}{l}\text { Inpatient } \\
\text { severe ( } N= \\
28)\end{array}$ & $\begin{array}{l}\text { P- } \\
\text { value }\end{array}$ \\
\hline Age (yrs) & $\begin{array}{l}8.22 \pm 6.78 \\
(334)\end{array}$ & $\begin{array}{l}5.6 \pm 6.27 \\
(293)\end{array}$ & & $5.69 \pm 6.3(265)$ & $4.68 \pm 6(28)$ & 0.416 \\
\hline $\operatorname{Sex}(F)$ & $162(49)$ & $137(47)$ & 0.662 & $119(45)$ & $18(64)$ & 0.051 \\
\hline \multicolumn{7}{|l|}{ Ethnicity } \\
\hline Hispanic or Latino & 64 (19) & $17(6)$ & & $15(6)$ & $\leq 10$ & 0.999 \\
\hline Not Hispanic or Latino & $68(20)$ & $68(23)$ & 0.388 & $55(21)$ & $13(46)$ & $0.002^{*}$ \\
\hline Unknown Ethnicity & $202(61)$ & $191(65)$ & 0.224 & $178(67)$ & $13(46)$ & $0.028^{*}$ \\
\hline \multicolumn{7}{|l|}{ Race } \\
\hline White & $137(41)$ & $109(37)$ & 0.329 & $96(36)$ & $12(46)$ & 0.288 \\
\hline Black or African American & $33(10)$ & $54(18)$ & $0.002^{*}$ & $46(17)$ & $8(29)$ & 0.231 \\
\hline Asian & $\leq 10$ & $\leq 10$ & - & $\leq 10$ & $0(0)$ & - \\
\hline American Indian or Alaskan Native & $0(0)$ & $0(0)$ & - & $0(0)$ & $0(0)$ & - \\
\hline Native Hawaiian or other Pacific Islander & $\leq 10$ & $0(0)$ & - & $0(0)$ & $0(0)$ & - \\
\hline Unknown Race & $157(47)$ & $144(49)$ & 0.592 & $137(52)$ & $7(25)$ & \\
\hline \multicolumn{7}{|l|}{ Co-morbidities } \\
\hline Congenital heart disease & $0(0)$ & $0(0)$ & - & $0(0)$ & $0(0)$ & - \\
\hline Other forms of heart disease & $20(6)$ & $41(14)$ & $0.001^{*}$ & $28(11)$ & $14(50)$ & \\
\hline Cystic Fibrosis & $(\leq 10)$ & $(\leq 10)$ & - & $\leq 10$ & $\leq 10$ & - \\
\hline
\end{tabular}




\section{Cureus}

\begin{tabular}{|c|c|c|c|c|c|c|}
\hline Asthma & 48 (14) & 37 (13) & 0.525 & $33(12)$ & $4(14)$ & 0.999 \\
\hline Premature lungs & $0(0)$ & $0(0)$ & - & $0(0)$ & $0(0)$ & - \\
\hline $\begin{array}{l}\text { Bronchopulmonary dysplasia originating in the } \\
\text { perinatal period }\end{array}$ & $(\leq 10)$ & $(\leq 10)$ & - & $\leq 10$ & $\leq 10$ & - \\
\hline Diseases of the Respiratory System & $217(65)$ & $162(55)$ & $0.013^{\star}$ & $140(53)$ & $24(86)$ & $0.001^{*}$ \\
\hline Neoplasms & $28(8)$ & $28(10)$ & 0.607 & $23(9)$ & $5(17)$ & 0.906 \\
\hline Overweight, obesity & $26(8)$ & $14(5)$ & 0.124 & $12(5)$ & $2(7)$ & 0.880 \\
\hline Diabetes insipidus & $0(0)$ & $(\leq 10)$ & - & $\leq 10$ & $\leq 10$ & - \\
\hline Diabetes mellitus & $(\leq 10)$ & $11(4)$ & - & $\leq 10$ & $\leq 10$ & - \\
\hline Endocrine, nutritional, and metabolic diseases & $73(22)$ & $85(29)$ & $0.039^{*}$ & $67(25)$ & $21(75)$ & \\
\hline Neuromuscular scoliosis & $(\leq 10)$ & $(\leq 10)$ & - & $\leq 10$ & $0(0)$ & - \\
\hline $\begin{array}{l}\text { Congenital malformations, deformations, and } \\
\text { chromosomal abnormalities }\end{array}$ & $58(17)$ & $78(27)$ & $0.005^{*}$ & $59(22)$ & $20(71)$ & \\
\hline Diseases of the nervous system & $72(22)$ & $77(26)$ & 0.166 & $60(23)$ & $20(71)$ & \\
\hline $\begin{array}{l}\text { Diseases of the musculoskeletal system and } \\
\text { connective system }\end{array}$ & $88(26)$ & $66(23)$ & 0.267 & $52(20)$ & $15(54)$ & \\
\hline Symptom at COVID-19 Diagnosis & & & & & & - \\
\hline Vomiting & $11(3)$ & $13(4)$ & 0.456 & $\leq 10$ & $\leq 10$ & \\
\hline Pain in joint & $0(0)$ & $(\leq 10)$ & - & $\leq 10$ & $0(0)$ & - \\
\hline Malaise and fatigue & $(\leq 10)$ & $14(5)$ & - & $\leq 10$ & $\leq 10$ & - \\
\hline Shortness of breath & $(\leq 10)$ & $(\leq 10)$ & - & $\leq 10$ & $0(0)$ & - \\
\hline Otalgia and effusion of ear & $(\leq 10)$ & $(\leq 10)$ & - & $0(0)$ & $0(0)$ & - \\
\hline Nasal congestion & $11(3)$ & $(\leq 10)$ & - & $\leq 10$ & $0(0)$ & - \\
\hline Myalgia & $0(0)$ & $(\leq 10)$ & - & $\leq 10$ & $0(0)$ & - \\
\hline Muscle weakness (generalized) & $0(0)$ & $(\leq 10)$ & - & $\leq 10$ & $0(0)$ & - \\
\hline Headache & $(\leq 10)$ & $(\leq 10)$ & - & $\leq 10$ & $0(0)$ & - \\
\hline Diarrhea, unspecified & $(\leq 10)$ & $20(7)$ & - & $16(6)$ & $4(14)$ & 0.211 \\
\hline Cough & $35(10)$ & 40 (14) & 0.272 & $33(12)$ & $7(25)$ & 0.121 \\
\hline Chills (without fever) & $(\leq 10)$ & $0(0)$ & - & $0(0)$ & $0(0)$ & - \\
\hline Anorexia & $0(0)$ & $0(0)$ & - & $0(0)$ & $0(0)$ & - \\
\hline Acute pharyngitis & $24(7)$ & $(\leq 10)$ & - & $\leq 10$ & $0(0)$ & - \\
\hline Fever of other and unknown origin & $42(13)$ & $77(26)$ & & $70(26)$ & $7(25)$ & 0.999 \\
\hline Dyspnea & $14(4)$ & $43(15)$ & & 29 (11) & $14(50)$ & \\
\hline \multicolumn{7}{|l|}{ Medications at COVID-19 Diagnosis } \\
\hline Glucocorticoids & $20(6)$ & $42(14)$ & $0.0005^{*}$ & $32(12)$ & $10(36)$ & $0.002^{*}$ \\
\hline Ibuprofen & $16(5)$ & $43(15)$ & & 37 (14) & $6(21)$ & 0.435 \\
\hline Ace inhibitors & $0(0)$ & $(\leq 10)$ & - & $\leq 10$ & $\leq 10$ & - \\
\hline Anticoagulants & $(\leq 10)$ & $26(9)$ & - & $21(8)$ & $5(18)$ & 0.159 \\
\hline Warfarin & $0(0)$ & $(\leq 10)$ & - & $\leq 10$ & $0(0)$ & - \\
\hline Remdesivir & $0(0)$ & $0(0)$ & - & $0(0)$ & $0(0)$ & - \\
\hline Hydroxychloroquine & $0(0)$ & $(\leq 10)$ & - & $\leq 10$ & $\leq 10$ & - \\
\hline
\end{tabular}




\section{Cureus}

\begin{tabular}{|c|c|c|c|c|c|c|}
\hline Chloroquine & $0(0)$ & $0(0)$ & - & $0(0)$ & $0(0)$ & - \\
\hline Azithromycin & $(\leq 10)$ & $13(4)$ & - & $11(4)$ & $2(7)$ & 0.804 \\
\hline Lopinavir/ritonavir & $0(0)$ & $0(0)$ & - & $0(0)$ & $0(0)$ & - \\
\hline Tocilizumab & $0(0)$ & $(\leq 10)$ & - & $0(0)$ & $\leq 10$ & - \\
\hline
\end{tabular}

\section{TABLE 1: Pediatric outpatient all vs. inpatient all and inpatient non-severe vs. inpatient severe}

Note: TriNetX software platform outputs all values $1: 10$ as $\leq 10$. Continuous values are followed by the number of included patients in parenthesis. Due to the obfuscation of patient numbers to keep the de-identification of data in the network, numbers are rounded thus creating differences within and between cohorts.

Mean \pm standard deviations

Number in parentheses reflect \% of participants in group

* represent $\mathrm{p}$ values $<0.05$

Pediatric patients ranged in age from $<1$ to 18 years, with a mean age of seven years, $48 \%$ were females. Race was unknown for $45 \%(n=282)$ of patients; of those known, $38 \%$ were white, $14 \%$ were African American, $2 \%$ were Asian. Ethnicity was known for $33 \%$ of patients and among those, $12 \%$ were Hispanic.

Inpatients were significantly younger than outpatients (mean age of 5.6 years vs 8.2 years, $p<0.001$ ), specifically, $61 \%$ of patients in the inpatient group were $<5$ years of age vs $44 \%$ in the outpatient group. There was no significant difference in sex between inpatient and outpatient groups $(\mathrm{p}=0.662)$. Among those for whom race was known, there was significantly more African American inpatients compared to outpatients ( $18 \%$ vs $10 \%, p=0.002$ ), but no difference for white participants $(\mathrm{p}=0.329)$. Among patients for whom ethnicity was known, there were more Hispanic or Latino outpatients compared to inpatients (19\% vs $6 \%, \mathrm{p}<0.001)$.

There was no significant difference between the groups for patients with a history of asthma (14\% outpatient, vs $13 \%$ inpatient, $\mathrm{p}=0.524$ ), neoplasms ( $8 \%$ outpatient vs $10 \%$ inpatient, $\mathrm{p}=0.607$ ) or history of obesity ( $8 \%$ outpatient vs $5 \%$ inpatient, $\mathrm{p}=0.123$ ). As shown in Table 1 , patients hospitalized were more likely to have a history of endocrine/metabolic disease ( $22 \%$ outpatient vs $29 \%$ inpatient, $p=0.039$ ), congenital malformations or chromosomal abnormalities ( $17 \%$ outpatient vs $27 \%$ inpatient, $\mathrm{p}=0.005$ ) as well as noncongenital heart disease ( $6 \%$ outpatient vs $14 \%$ inpatient, $p<0.001)$.

Common symptoms recorded for outpatients at time of COVID-19 diagnosis included fever and cough in $13 \%$ and $10 \%$, respectively. Other symptoms include dyspnea (4\%), pharyngitis (7\%), nasal congestion (3\%), and vomiting (3\%). Less than $3 \%$ of subjects had malaise/fatigue, headache, ear complaints, diarrhea, or chills. In contrast, $26 \%$ of inpatients had fever reported at time of diagnosis, $15 \%$ with dyspnea, $14 \%$ with cough, $7 \%$ with diarrhea, $5 \%$ with malaise/fatigue, and $4 \%$ with vomiting. Less than $3 \%$ of subjects reported nasal congestion, headache, pharyngitis, chills, or ear complaints. Significantly more inpatients had fever and dyspnea $(\mathrm{p}=0.001)$ at the time of COVID-19 presentation.

In terms of laboratory values, few outpatients had laboratory evaluation recorded. More values were recorded for inpatients. Several laboratory values were significantly more elevated in inpatients including: mean lactate dehydrogenase (LDH) (396 vs. 270 units/L, $\mathrm{p}=0.018$ ), mean C reactive protein (CRP) (23.7 vs. $4.56 \mathrm{mg} / \mathrm{L}, \mathrm{p}=0.044$ ) and mean amylase (143 vs $38 \mathrm{U} / \mathrm{L}, \mathrm{p}-0.045)$.

Less than $3 \%$ of outpatients were given azithromycin, but none were reported to have remdesivir, hydroxychloroquine/chloroquine, lopinavir/ritonavir, or tocilizumab. Among inpatients, $4 \%$ were given azithromycin, none were given remdesivir, and less than $3 \%$ received hydroxychloroquine. Tocilizumab was given to $<10$ inpatients and $9 \%$ of inpatients were anticoagulated. Significantly more inpatients received ibuprofen and glucocorticoids $(\mathrm{p}<0.001)$.

\section{Inpatients non-severe vs. severe}

Amongst 293 inpatients, 90\% ( $n=265)$ were non-severe and 10\% $(n=28)$ were classified as severe (Table 1). Of the severe inpatients, 27 were mechanically ventilated and one patient died.

Overall there was no significant age difference, however, the percentage of patients $<5$ years was higher in severe inpatients vs. non-severe ( $71 \%$ vs $60 \%$ ) (Figure 1 ). 


\section{Cureus}

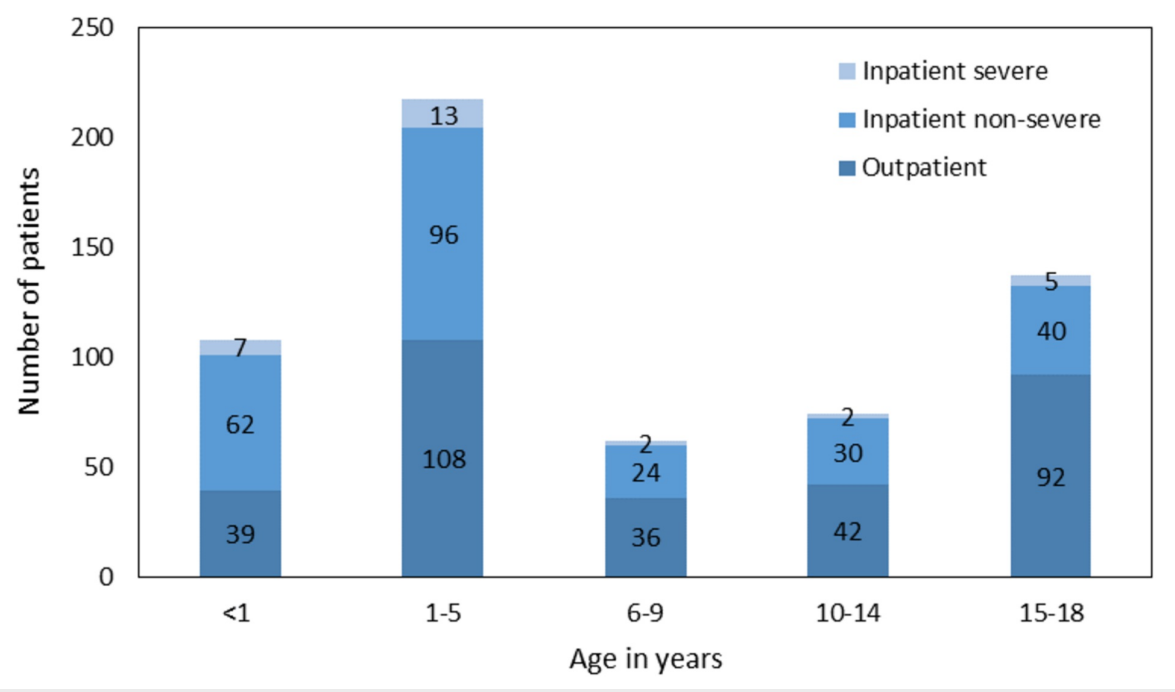

\section{FIGURE 1: Hospitalization status and severity by age group}

Note: Numbers represent actual number of patients in each age group.

Actual age is missing for about 15-20 patients in the cohort. This can be explained by one or few healthcare organizations contributing patients under 18 to the network but not disclosing actual age.

Although not significantly different, there was a trend towards more females classified as being severe (64\% severe vs $45 \%$ non-severe, $\mathrm{p}=0.051$ ). Of those with known ethnicity, significantly more severe patients were not Hispanic or Latino ( $46 \%$ vs $21 \%$, p=0.002). There was no significant differences for those with known race.

Significantly more patients with a severe illness had a history of co-morbidity including non-congenital heart disease ( $50 \%$ vs $11 \%$, p $<0.001)$, disease of the respiratory system ( $86 \%$ vs $53 \%$, p < 0.001 ) metabolic disease/endocrine disease ( $75 \%$ vs $25 \%$, p<0.001), congenital malformation or chromosomal abnormalities ( $71 \%$ vs $22 \%$, p <0.001), and disease of the nervous system ( $71 \%$ vs $23 \%$, p<0.001). Similar trends were seen among patients less than five years of age, more patients in the severe category had a history of co-morbidity (data not shown).

The severe patients were more likely to have dyspnea $(\mathrm{p}<0.001)$.

The severe inpatient group had significantly elevated prothrombin time ( $15.9 \mathrm{vs} 13.6$ seconds, $\mathrm{p}=0.028$ ) and activated partial thromboplastin time ( 36.5 vs 29.2 seconds, $\mathrm{p}=0.018$ ). Additionally, alanine aminotransferase and aspartate aminotransferase were also higher in the severe inpatient group compared to non-severe, 268 vs. $43.9 \mathrm{U} / \mathrm{L}$ ( $\mathrm{p}=0.003$ ) and 212 vs $55.6 \mathrm{U} / \mathrm{L}(\mathrm{p}=0.008)$, respectively.

Azithromycin was given to 11 (4\%) of the non-severe inpatients and two severe inpatients. Tocilizumab was only given to severe inpatients $(n \leqslant 10)$. Steroids were more likely to be given to severe inpatients $(36 \%$ vs $12 \%, \mathrm{p}=0.002)$. Anticoagulation was recorded for $26(9 \%)$ inpatients, 21 were non-severe, and five were categorized as severe.

\section{Discussion}

At the time of submission, there was limited published data on clinical characteristics of pediatric patients with COVID-19 in the United States from large health care organizations. Our findings add to the current knowledge of clinical presentation and care of COVID-19 in children in the outpatient and inpatient settings.

In our analysis, a higher proportion of pediatric COVID-19 cases were hospitalized when compared to prior reports reported [5-6,9]. However, consistent with our current knowledge, more severe clinical presentations of COVID-19 were found in younger children under the age of five years requiring hospitalizations. Patients with an underlying condition were more likely to require hospitalization and have a severe presentation.

Global data suggest that there is a sex difference in mortality from COVID-19 [10]. In a preliminary analysis from the CDC, $57 \%$ of COVID-19 pediatric cases were males [5], and similarly slightly more males than females were affected in reported pediatric data from China [6]. In our study, there was no significant sex differences between patients requiring hospitalization, however, once hospitalized there was a trend for 
increased severity in females. This supports that sex differences are likely multi-factorial and may include sex hormone-influenced mechanisms as well as gender-based behavioral factors which may not become relevant until puberty.

Persons who are African American or black have higher mortality in the US, with an infection rate more than three-fold higher in predominantly black counties compared to predominantly white counties [11]. In our analysis, we found that there was significantly more African American inpatients compared to outpatients. Our study design does not allow us to adjust for comorbidities, however, we highlight that racial differences in COVID-19 disease severity which may be due to health care disparity extend to the pediatric population.

Outpatients reported the characteristic COVID-19 symptoms of fever and cough, however, more severe patients requiring hospitalization had fever and dyspnea. Very few patients presented with diarrhea or vomiting, unlike what has been reported for patients with Multisystem Inflammatory Syndrome in Children (MIS-C) [12]. Initial coagulopathy of COVID-19 in adult patients typically presents with elevation of D-dimer and fibrin degradation products, while abnormalities in prothrombin time and partial thromboplastin time are uncommon [13]. Our findings support the prevalence of activation of the coagulation cascade in the pediatric population with a severe presentation. This is significant as it is often predictive of a poor outcome or high mortality [14].

We found that few pediatric patients are receiving azithromycin and hydroxychloroquine, and those hospitalized with severe presentation are more likely to be given tocilizumab and anticoagulants.

This study has several strengths. To our knowledge, this is the first report from a large research network database with a focus on pediatric COVID-19 allowing us to have a more comprehensive picture of inpatient vs outpatient pediatric presentation. This study also has a number of limitations. Due to the nature of the database, we were unable to collect patient level data on specific outcomes and prognosis and duration of illness. We were unable to report on radiology information. We also were unable to report respiratory support needed such as nasal cannula, invasive or non-invasive ventilation, or on impact of any treatments given. We do not have information on type of diagnostic test used for confirmation of disease, whether nasopharyngeal swab vs. antibody testing vs. viral testing from other location (such as rectal swab). We were unable to report on source of transmission, whether travel related, community-acquired, or household source. Lastly, we do not have long-term follow up data on these patients. We do not know whether any of these patients had symptoms consistent with MIS-C after the initial COVID presentation.

\section{Conclusions}

The number of COVID-19 cases continue to increase and our study suggests that clinicians should remain vigilant when monitoring young children with underlying conditions and COVID-19, as they may be more likely to be hospitalized and have a higher severity of disease. Studies on transmission of disease, clinical course, treatment, and prognosis are needed in pediatrics. Long term follow-up of these patients are needed to understand late manifestations of the disease, including MIS-C.

\section{Additional Information \\ Disclosures}

Human subjects: Consent was obtained by all participants in this study. Animal subjects: All authors have confirmed that this study did not involve animal subjects or tissue. Conflicts of interest: In compliance with the ICMJE uniform disclosure form, all authors declare the following: Payment/services info: This publication was made possible through funding support of University Hospitals Cleveland Medical Center (UHCMC) and the Clinical and Translational Science Collaborative of Cleveland, UL1TR002548 from the National Center for Advancing Translational Sciences (NCATS) component of the National Institutes of Health and NIH roadmap for Medical Research. Its contents are solely the responsibility of the authors and do not necessarily represent the official views of UHCMC or the NIH. Financial relationships: Grace A. McComsey declare(s) a grant and personal fees from Merck, Gilead, Viiv, Theratechnologies, Roche, Astellas, Tetraphase, BMS. Dr. McComsey served as a consultant for Merck, Gilead, Viiv, and Theratechnologies, and has received research funding from Roche, Astella, Tetraphase, and BMS. Other relationships: All authors have declared that there are no other relationships or activities that could appear to have influenced the submitted work.

\section{Acknowledgements}

This publication was made possible through funding support of University Hospitals Cleveland Medical Center (UHCMC) and the Clinical and Translational Science Collaborative of Cleveland, UL1TR002548 from the National Center for Advancing Translational Sciences (NCATS) component of the National Institutes of Health and NIH roadmap for Medical Research. Its contents are solely the responsibility of the authors and do not necessarily represent the official views of UHCMC or the NIH.

\section{References}


1. Castagnoli R, Votto M, Licari A, et al.: Severe acute respiratory syndrome coronavirus 2 (SARS-CoV-2) infection in children and adolescents: a systematic review. JAMA Pediatr. 2020, 174:882-889.

10.1001/jamapediatrics.2020.1467

2. Hasan A, Mehmood N, Fergie J: Coronavirus disease (COVID-19) and pediatric patients: a review of epidemiology, symptomatology, laboratory and imaging results to guide the development of a management algorithm. Cureus. 2020, 12:e7485. 10.7759/cureus.7485

3. Zhu L, Lu X, Chen L: Possible causes for decreased susceptibility of children to coronavirus . Pediatr Res. 2020, 88:342. 10.1038/s41390-020-0892-8

4. Zimmermann P, Curtis N: Coronavirus infections in children including COVID-19: an overview of the epidemiology, clinical features, diagnosis, treatment and prevention options in children. Pediatr Infect Dis J. 2020, 39:355-68. 10.1097/INF.0000000000002660

5. CDC COVID-19 Response Team: Coronavirus disease 2019 in children - United States, February 12-April 2, 2020. MMWR Morb Mortal Wkly Rep. 2020, 69:422-6. 10.15585/mmwr.mm6914e4

6. Dong Y, Mo X, Hu Y, Qi X, Jiang J, Jiang Z, Tong S: Epidemiology of COVID-19 among children in China . Pediatrics. 2020, 145:e20200702. https://doi.org/10.1542/peds.2020-0702

7. Yagnik PJ, Umscheid J, Khan AW, Ali M, Bhatt P, Desai PH: Pediatric characteristics of 2019 novel coronavirus: review of available published literature. Clin Pediatr. 2020, 59:849-852. $10.1177 / 0009922820920017$

8. Chiotos K, Hayes M, Kimberlin DW, et al.: Multicenter initial guidance on use of antivirals for children with coronavirus disease 2019/severe acute respiratory syndrome coronavirus 2. J Pediat Inf Dis Soc. 2020, piaa045. 10.1093/jpids/piaa045

9. Lu X, Zhang L, Du H, et al.: SARS-CoV-2 infection in children. N Engl J Med. 2020, 382:1663-5. 10.1056/NEJMc2005073

10. Sharma G, Volgman AS, Michos ED: Sex differences in mortality from COVID-19 pandemic: are men vulnerable and women protected?. JACC Case Rep. 2020, 2:1407-1410. 10.1016/j.jaccas.2020.04.027

11. Yancy CW: COVID-19 and African Americans. JAMA. 2020, 323:1891-1892. 10.1001/jama.2020.6548

12. Chiotos K, Bassiri H, Behrens E, et al.: Multisystem inflammatory syndrome in children during the coronavirus 2019 pandemic: a case series. J Pediatric Infect Dis Soc. 2020, 9:393-398. 10.1093/jpids/piaa069

13. Connors JM, Levy JH: COVID-19 and its implications for thrombosis and anticoagulation. Blood. 2020, 135:2033-2040. 10.1182/blood.2020006000

14. Klok FA, den Exter PL, Huisman MV, Eikenboom J: Dealing with COVID-19-associated coagulopathy. Ned Tijdschr Geneeskd. 2020, 164: 